\title{
Pratiques
}

Linguistique, littérature, didactique

173-174| 2017

Le déjà-là dans l'écriture

\section{La novélisation : une rencontre avec du déjà-là qui révèle les positionnements auctoriaux des jeunes scripteurs}

Novelization as a way of revealing pupils' auctorial positions

\section{Sonia Castagnet-Caignec}

\section{OpenEdition}

\section{Journals}

Édition électronique

URL : http://journals.openedition.org/pratiques/3395

DOI : 10.4000/pratiques.3395

ISSN : 2425-2042

Éditeur

Centre de recherche sur les médiations (CREM)

Référence électronique

Sonia Castagnet-Caignec, « La novélisation : une rencontre avec du déjà-là qui révèle les positionnements auctoriaux des jeunes scripteurs », Pratiques [En ligne], 173-174 | 2017, mis en ligne le 10 mars 2017, consulté le 04 mai 2019. URL : http://journals.openedition.org/pratiques/3395 ; DOI : 10.4000/pratiques.3395

Ce document a été généré automatiquement le 4 mai 2019.

(c) Tous droits réservés 


\title{
La novélisation : une rencontre avec du déjà-là qui révèle les positionnements auctoriaux des jeunes scripteurs
}

Novelization as a way of revealing pupils' auctorial positions

\author{
Sonia Castagnet-Caignec
}

L'école a depuis longtemps cherché à développer les capacités d'écriture de ses élèves au travers d'exercices d'écriture littéraire qui se réfèrent, pour simplifier, soit à des pratiques d'imitation soit à des pratiques dites d'imagination ou d'invention dont les modèles ne sont évidemment pas absents. Ainsi, la scolarisation des genres littéraires est un phénomène attesté dans les classes et dans les pratiques enseignantes et le genre scolaire se distingue quelque peu de son homologue littéraire de par son traitement didactique en classe et sa reconstruction auprès des élèves via le prisme de l'enseignement-apprentissage. Il s'avère alors utile de soumettre les élèves à une pratique d'écriture autour d'un genre encore neutre dans l'univers scolaire pour observer les compétences qu'ils démontrent, les obstacles qu'ils rencontrent et les différentes habiletés qu'ils développent au cours de cet exercice relativement original pour eux. Écrire à partir d'un film avec en perspective la réalisation d'un écrit à visée littéraire, tel que le veut la novélisation, nous renseigne sur les différentes procédures mises en œuvre par les élèves. En effet, la novélisation reste un objet relativement méconnu des élèves et de l'école, non exploité malgré ses potentialités en termes de mobilisation du déjà-là filmique et de la libération qu'elle offre au scripteur au niveau d'une partie de la planification. L'enjeu sera donc d'observer comment les élèves d'âges contrastés manipulent ce genre dans leur production et comment ce genre permet de repérer des profils d'élèves. 


\section{Retour sur le genre}

\subsection{Bref panorama}

2 La novélisation constitue un genre littéraire ou paralittéraire qui émerge de façon quasi simultanée avec les débuts du cinéma. Cette pratique consiste en l'adaptation d'un récit filmique en récit écrit et a pour vocation première de prolonger le plaisir de la fiction filmique dans celui de la lecture grâce à une communauté d'univers diégétiques jusqu'à viser même la duplication parfaite du film au travers du livre. Cette pratique s'épanouit évidemment dans une époque où les films ne bénéficient pas de la même facilité d'accès qu'actuellement: diffusion exclusive en salle, roulement des copies qui contraint les circuits de diffusion, etc. Ainsi dès le début du $\mathrm{XX}^{\mathrm{e}}$ siècle, des périodiques proposent des récits écrits de films illustrés de photographies : Le film complet à partir de novembre 1922, Mon film, Ciné-Miroir, etc. sans compter la vogue des publications de feuilletons vers 1915 dans les quotidiens nationaux (Le matin par exemple), feuilletons souvent issus de films à épisodes. On dénombre aussi dans ces pratiques, dès 1910, les programmes payants de films qui accompagnaient la projection et qui racontaient le film ${ }^{1}$. Ces romans-cinéma permettent ainsi aux spectateurs de revivre l'expérience esthétique et/ou récréative du visionnage « unique » du film en salle et ce, à un prix modique. Cette pratique non encore baptisée novélisation va revêtir des formes très diverses au cours du temps et de l'histoire du cinéma pour apparaître sous un format plus ou moins stabilisé dans les années 1980 sous le nom de novélisation (roman adapté d'un film ou d'un scénario selon Le Petit Robert, édition 1995), avec un nombre assez conséquent de titres publiés chez J'ai lu et Pocket. Cependant, les manifestations du genre demeurent hybrides et tributaires des intentions des différents auteurs ainsi que de leur inscription dans les champs de la création artistique et littéraire : on assiste ainsi à la production quasi massive et industrielle de novélisations dans les célèbres collections Bibliothèque Rose et Verte chez Hachette - ces dernières s'emparent des séries animées plébiscitées à la télévision - et, simultanément, à des expériences esthétiques inédites telles que celles de Tanguy Viel dans son ouvrage Cinéma (1999). Ce livre sous-titré roman s'apparente à une adaptation libre et très personnelle à l'écrit de Sleuth (Le limier) de J. L. Mankiewicz - considéré par certains spécialistes comme une sorte de remake écrit du film - plutôt qu'à une novélisation "classique». Il est pourtant notable que la circularité des œuvres observée dans les débuts du cinéma et la perméabilité des supports (cinéma, théâtre, feuilletons, livres, revues...) se rapproche très considérablement des pratiques actuelles d'emprunt et d'échanges d'œuvres entre médias et rejoint les habitudes contemporaines de transposition. Quelques exemples actuels sont frappants: Ils ne sont pour rien dans mes larmes d'olivia Rosenthal (2012) est d'abord le récit écrit d'expériences cinématographiques diverses, l'épilogue Les Larmes est ensuite adapté en court métrage éponyme tandis que le prologue Le Vertige l'est en spectacle théâtral, tous deux faisant respectivement référence au film de Jacques Demy Les Parapluies de Cherbourg et à Vertigo d'Alfred Hitchcock ; la fameuse Guerre des boutons de Louis Pergaud (1981) fut adaptée par Yves Robert et François Boyer en un film non moins célèbre d'Yves Robert en 1962, un demi-siècle plus tard, deux remakes du film sortent simultanément en 2011 dont l'un fait l'objet d'une novélisation de Nicolas Jaillet sous-titrée « le roman du film ». 
3 Si l'on considère le versant plus commercial de la novélisation, l'investissement de celleci par des ouvrages en série de la littérature jeunesse depuis le début des années 2000 a de fait permis un regain de visibilité et d'intérêt pour cette forme générique assez peu reconnue tant par le public que par les instances littéraires (critiques ou études). On voit ainsi émerger quelques contributions sur le genre (Baetens, 2006 et 2008; Ferrier, 2006; Bourhis, Le Corff, 2013) qui interrogent le statut de celui-ci. Les auteurs cités envisagent le phénomène du point de vue de la réception de ces œuvres atypiques, du rapport entre cinéma/littérature, éventuellement dans la perspective d'une approche nouvelle des pratiques de lecture des enfants.

\subsection{Quelques éléments de cadrage théorique pour définir le genre}

4 Pour dépasser la simple dichotomie entre littérature commerciale/paralittérature et novélisation dite « littéraire », nous allons tenter de clarifier le statut du genre du point de vue de son organisation discursive et de sa proximité avec le fonctionnement des textes littéraires.

\subsubsection{Intertextualité (ou interfilmicité ?)}

5 La relation de dépendance au film dans la novélisation n'est pas sans rappeler les phénomènes d'intertextualité telle que définie originellement par Julia Kristeva (1969) et surtout d'hypertextualité (Genette, 1982) si courants en littérature, à la différence près qu'ici la référence intertextuelle est constituée d'un film non d'un texte. Nous pouvons également y discerner une approche intertextuelle plus récente relevant à la fois du principe de la citation pure (le film-source) et de celui de la référentialité en dehors du champ strictement littéraire (Compagnon, 1979 et 1998).

De fait, il est entendu que le cinéma est une langue à part entière (Metz, 1968) comportant sa propre grammaire et son propre système sémiotique - dans lequel les signes deviennent des kinèmes ou cinènes (Pasolini, 1966). Ainsi langage cinématographique et langage écrit se rencontrent au carrefour de la novélisation dans une relation d'interdépendance qui nous amène à définir le film comme l'hypotexte (Genette, 1982) du récit novélisé lui-même hypertexte du film. Dans les cas typiques de novélisation, les phénomènes de réécriture et d'adaptation de l'hypotexte-film se contentent de traduire au «premier degré " sans véritable recherche d'équivalences entre les deux codes sémiotiques différents: le récit novélisé transcrit uniquement la matière narrative filmique en matière narrative écrite en respectant la bible du film. On ne peut parler d'une recherche particulière inhérente au passage d'une langue à une autre telle qu'on peut la trouver dans les novélisations au second degré (Baetens, 2006) qui se démarquent de la production globale ${ }^{2}$. Le texte novélisé assume la fonction de fixer le film, voire de le figer à l'écrit pour dépasser l'évanescence de l'image.

\subsubsection{Novélisation et mimesis}

7 Cette fonction de traduction mais surtout de fixation inscrit le genre dans la tradition aristotélicienne de la mimesis : la novélisation a pour charge de reproduire le film, de rendre compte du film par écrit, de substituer les mots aux images et en quelque sorte de les dupliquer. La novélisation imite l'image qui imite elle-même le réel. Ici le double miroir fonctionne comme un filtre qui peut agir a contrario de l'effet de réel recherché 
dans le texte écrit: en effet, concevoir la novélisation comme le texte qui sous-titre le récit filmique expose le discours écrit à une réduction à la fois de l'œuvre-source mais également à un appauvrissement intrinsèque en ne répondant pas aux critères de recherche formelle et stylistique inhérents au texte littéraire. Ainsi, la novélisation est sujette à la déformation, à la simplification, à la linéarisation et à l'explication d'un langage cinématographique complexe et abouti en lui-même qui fait déjà œuvre et sens. La novélisation tient grandement de cette volonté de percer le mystère et l'implicite du récit filmique, elle est notamment le lieu de l'explicitation des intentions, des états psychologiques des personnages non élucidés par le récit filmique, le terrain idéal du déploiement des non-dits entre personnages, des discours intérieurs projetés sur les personnages. Et c'est là que se jouent parallèlement sa fonction et son désaveu : en désépaississant la complexité filmique, elle se voit sous-classée en littérature.

\subsubsection{Novélisation et genre de discours second}

Pourtant, nous placerons résolument la novélisation au rang de genre de discours second au sens bakhtinien (Bakhtine, 1984). En effet, la novélisation répond à un type relativement stable d'énoncé comportant des unités thématiques et compositionnelles déterminées (ibid., p. 266, 269) : il s'agit bien de la retranscription du discours filmique en discours écrit sous la forme d'un roman en respectant les fonctions de traduction, linéarisation, et fixation.

Les épiphénomènes cités plus haut (novélisations au second degré) renvoient à des choix stylistiques propres, à un style individuel (ibid., p. 268) qui participent de la variété du genre organisé autour d'un champ homogène et standardisé (celui de la novélisation dite commerciale) qui permet néanmoins de fonder le genre comme genre de discours second grâce à la récurrence et à la stabilité du fonctionnement des énoncés. Ainsi les postulats de M. Bakhtine nous permettent d'évacuer les considérations qualitatives sur des œuvres prises isolément, au profit d'une analyse plus englobante du phénomène. Les novélisations s'inscrivent bien comme genre de discours second au-delà des suspicions et des a priori sur sa légitimité et sa littérarité à l'intérieur d'une sphère d'échange verbal qui est celle de la littérature et de sa réception, littérature de jeunesse et paralittérature y comprises.

\subsection{La novélisation : un genre du déjà-là par excellence ou...}

\subsubsection{Par essence}

On l'a vu, la novélisation est par essence un type d'écriture de référenciation à une source antérieure, tributaire de l'hypotexte filmique, dans la lignée du dialogisme bakhtinien intradiscursif au sens où « [l'expression de l'énoncé] manifeste non seulement son propre rapport à l'objet de l'énoncé mais aussi le rapport du locuteur aux énoncés d'autrui » (ibid., p. 299). Dans le cas de la novélisation, l'objet de l'énoncé est de surcroît « un texte antérieur » (l'hypotexte filmique) avec lequel l'auteur a noué une forme de familiarité - et de dialogue - qui peut s'envisager comme un rapport particulier à cet énoncé-film. La réponse au film que constitue l'énoncé-novélisation intègre forcément le point de vue d'autrui, elle constitue une réaction à cet énoncé autre et en cela participe des harmoniques dialogiques (ibid., p. 300) dans un discours que l'on peut, à ce titre, qualifier de polyphonique tant les sphères d'échange verbal et culturel sont complexes et entrelacées dans 
cette relation duelle film-texte. C'est en partie dans cette réponse au film et dans la négociation de l'énoncé d'autrui, dans sa transformation que réside l'enjeu de la novélisation et c'est peut-être bien parce que la transformation est minimale et minimaliste que la novélisation n'émerge pas en tant que genre littéraire, notamment aux yeux de la critique.

11 Pourtant, le tribut dû au film place indubitablement la novélisation dans la longue tradition des réécritures, des variations à partir d'un substrat originel. Nous nous risquerons à avancer que c'est justement cette relation revendiquée d'assujettissement au récit filmique qui entrave l'autonomie de la novélisation en tant que genre à part entière. En effet, dans cette dépendance complètement assumée d'hypotexte-film/hypertextenovélisation, l'ouvrage novélisé ne fait ni œuvre nouvelle ni œuvre indépendante mais ne prend son sens que dans le recours au film par le principe de la fixation mentionné plus haut. Ce parti-pris de fidélité au déjà-là filmique, cette opération de transposition par simple reformulation d'un type de discours à l'autre (filmique/écrit) participe en partie de la non-reconnaissance du genre. La novélisation ne fait pas preuve d'originalité, d' inventio mais copie, plagie le film. Les seuls processus de réduction ou d'amplification du récit filmique ne peuvent suffire à faire de l'ouvrage novélisé un objet autonome qui développerait son propre langage: ils ne sont que des moyens au service d'une retranscription du discours filmique. Le principe de la relinéarisation de la trame narrative du film dans la novélisation amène à reconstituer l'ordre logique et chronologique du film dans une continuité narrative "classique » alors que le film, lui, peut souffrir de télescopages temporels grâce au montage qui alterne des plans temporels simultanés ; cette simplification ne constitue qu'un exemple de paramètre spécifique au langage filmique non investi par le langage écrit.

\subsubsection{Par défaut}

Qui dit simplification dit éventuellement production décevante quant à l'horizon d'attente du lecteur (Jauss, 1978). Là encore, le critique littéraire ou le lecteur peuvent juger sévèrement le récit novélisé au regard des qualités développées dans le discours filmique initial, riche d'un langage complexe comme nous le signalions. L'adaptation se résume alors à un transfert de médium mais ne se réalise pas comme une réflexion et une pénétration d'un univers médiatique dans un autre. Pour se référer à un concept relativement moderne et en vogue, il s'agirait d'une intermédialité ${ }^{3}$ cette fois non totalement assumée en contraste avec la dépendance assumée au film: la relation filmtexte est univoque dans le cas de la novélisation, les processus intermédiatiques ne relèvent pas de l'interaction mais d'une relation de citation descendante du film au texte. Il s'agit d'un passage d'un support à l'autre commandé par les enjeux premiers de réception de la novélisation: conserver la trace écrite de l'histoire du film. Or l'intermédialité supporte des niveaux plus complexes de relation même si elle se réfère, en partie, théoriquement aux relations intertextuelles mises à jour en littérature et donc à des formes parfois minimes de dépendance. Les propos de P. Pasolini de 1966 résonnent ici étrangement en considération de leur diachronie par rapport au concept d'intermédialité: il esquissait déjà cette synthèse difficile entre les deux médias. Il soutenait ainsi que les kinèmes étaient irréductibles aux signes du discours écrit et que le scénario (que l'on peut considérer dans cette démonstration comme la figure de la novélisation) était un objet entre deux langages, un objet impossible qui n'atteint son aboutissement ni dans la forme du langage écrit ni dans celle du langage 
cinématographique. La novélisation se situe aussi dans cet entredeux, entre citation et référence, coprésence et transfert mais n'achève pas vraiment l'hybridation des deux médias.

\subsubsection{Par des pratiques culturelles et sociales}

Dans la continuité de ce qui précède, il est malgré tout utile de rappeler que la novélisation s'érige comme genre du déjà-là dans la contemporanéité des pratiques culturelles. Qu'il y ait ou non un degré élevé d'accomplissement d'intermédialité, il y a bel et bien dans la réception, l'usage et la pratique de ce genre littéraire par l'utilisateur actuel des médias, une circulation d'un média à l'autre, un décloisonnement de sa représentation des univers scripturaux et iconographiques qui le font sans cesse traverser les sphères de diffusion en passant du livre au film, du film au jeu vidéo, de la série au livre, etc. dans une modalité de réception nouvelle, liée notamment au mode de diffusion sur écran. Il est notable que le genre novélisation trouve son prolongement dans une pratique amateure d'écriture en ligne : les fanfiction. Ces sites sont alimentés par des aficionados de séries (ou films) qui inventent des suites inédites ou des variantes à des épisodes en respectant l'univers de la série. Le principe de la novélisation y est remarquablement présent : renouveler, prolonger le plaisir du film ou de la série - donc du langage filmique - au travers d'un discours écrit. Nous assistons ici à l'épanouissement d'une forme moderne du roman-cinéma du film muet. Le déjà-là filmique est la source du texte, son inspiration et même son mobile.

\section{2. Élaboration du corpus de recherche et expérimentation}

14 L'expérimentation ici décrite vise à observer les voies d'investissement du genre par des élèves d'un échantillon relativement large. Nous souhaitons ainsi déterminer les processus d'écriture que les élèves mettent en œuvre pour traiter la novélisation c'est-àdire pour transformer le système diégétique filmique en système diégétique écrit et créer un univers fictionnel suffisamment étoffé pour que le lecteur y souscrive. La question de départ se fonde évidemment sur la nouveauté de l'exercice pour ces élèves et le relatif non usage de ce type d'écrit en classe. Nous supposons que des éléments non observés antérieurement apparaîtront dans un recueil de productions d'élèves soumis à cette pratique peu courante d'écriture qui constitue un mélange de liberté et de contraintes, sans forme véritablement prescrite. Ce type d'écrit offre donc un terrain propice pour observer les comportements scripturaux des élèves et identifier la manière dont ils se positionnent comme auteurs à partir de l'observation de leurs procédures.

\subsection{Population étudiée}

Centre trente-huit élèves relevant du système français ont participé à l'expérimentation allant du CE1 de l'école primaire à la classe de première du niveau secondaire. Des classes comprenant entre 21 et 29 élèves se sont prêtées au jeu de la novélisation (une classe de CE1-CE2, deux classes de CM1-CM2, une classe de $5^{\mathrm{e}}$, une classe de $3^{\mathrm{e}}$, une classe de $1^{\mathrm{re}} \mathrm{L}$ ). Le bassin de recrutement de l'école primaire et du collège est le même (même zone géographique et même carte scolaire, zone classée péri-urbaine selon la nomenclature 
INSEE). Le recueil du lycée s'est fait en milieu urbain mais dans le même département (lycée de centre-ville, dans une section littéraire engagée dans le prix Renoir donc « familiarisée » à la critique de film).

Dans le cadre du présent article, nous nous concentrerons uniquement sur deux niveaux spécifiques et contrastés : les élèves en fin de primaire (29 élèves de CM1-CM2) et les élèves en fin de cursus secondaire (29 élèves de première littéraire). Au total, 349 productions ont été recueillies dont 111 seront étudiées ici.

\subsection{Protocole suivi}

Lors d'une première phase du protocole, le plus grand nombre des élèves de l'échantillon a visionné en situation écologique mais sans dispositif didactique deux séquences filmiques à novéliser à une semaine d'intervalle l'une de l'autre. Lors d'une deuxième phase du protocole, un nombre plus restreint d'élèves a bénéficié d'une séquence didactique composée de dix séances et articulée autour du genre comme outil didactique afin de les accompagner dans l'exercice de novélisation. en tant que révélateurs de tendances spontanées des élèves. La consigne qui a été fournie aux élèves de cette première phase était simple: immédiatement après la projection, ils devaient rédiger un écrit littéraire à partir du film, comme le ferait un écrivain. Les élèves visionnaient donc les séquences «comme au cinéma », une seule fois, sans prise de notes ou écriture, sans retour oral sur le film ni commentaires entre eux ou avec l'enseignant.

21 Il a d'abord été choisi un extrait du film de Jacques Tati Les Vacances de Monsieur Hulot. Une semaine plus tard, les élèves ont visionné dans son intégralité le court métrage Le Génie de la boîte de raviolis, film d'animation de Claude Barras, d'une durée quasi-équivalente à la séquence précédente. Ils ont ainsi été soumis au même exercice dans les mêmes conditions.

22 À l'issue de l'écriture de chacune des deux passations, les élèves ont renseigné un questionnaire sur les difficultés ou l'absence de difficultés à réaliser l'exercice.

\subsection{Analyse des supports choisis}

\subsubsection{Caractéristiques générales des deux supports filmiques}

Le tableau comparatif suivant permet d'identifier rapidement les traits généraux des séquences filmiques choisies.

Tableau 1. Caractéristiques générales des supports de l'expérimentation

\begin{tabular}{|l|l|l|}
\hline & Les vacances de monsieur Hulot & Le génie de la boîte de raviolis \\
\hline réalisation & Jacques Tati & Claude Barras \\
\hline année & 1953 (année de la première sortie) & 2006 (production 2005) \\
\hline
\end{tabular}




\begin{tabular}{|c|c|c|}
\hline durée & $\begin{array}{l}\text { extrait visionné : 7’34 (durée intégrale du } \\
\text { film : } 95 \text { minutes) }\end{array}$ & 7’30 visionné en intégralité \\
\hline technique & $\begin{array}{l}\text { Film en noir en blanc, sans dialogue, } \\
\text { proche du muet }\end{array}$ & $\begin{array}{l}\text { Film d'animation en couleurs, en } \\
\text { volume et trois dimensions avec } \\
\text { marionnettes }\end{array}$ \\
\hline genre & $\begin{array}{l}\text { Comédie (humour, film à sketchs } \\
\text { présentant des séquences relativement } \\
\text { autonomes et un découpage qui facilitent } \\
\text { le choix d'un extrait « isolé » du reste du } \\
\text { film mais cependant compréhensible) }\end{array}$ & $\begin{array}{l}\text { Comédie (merveilleux, conte } \\
\text { moderne, narratif) }\end{array}$ \\
\hline résumé & $\begin{array}{l}\text { Passage visionné par les élèves: } \\
\text { (la leçon d'équitation) } \\
\text { Monsieur Hulot, personnage très } \\
\text { maladroit, souhaite faire de l'équitation } \\
\text { en compagnie de Martine, jolie jeune fille } \\
\text { en vacances au bord de mer tout comme } \\
\text { lui. Hulot se rend au lieu de villégiature } \\
\text { de Martine et crée le plus grand désordre } \\
\text { dans le salon bourgeois où il l'attend. } \\
\text { Puis, sur la plage, monsieur Hulot tente } \\
\text { d'apprivoiser sa monture mais n'y } \\
\text { parvient pas. En revanche, il provoque un } \\
\text { enchaînement de catastrophes qui le } \\
\text { pousse à s'enfuir subrepticement vers sa } \\
\text { chambre d'hôtel. Cet abandon ne laisse } \\
\text { d'autre choix à la jeune femme que de } \\
\text { partir en promenade avec un autre } \\
\text { compagnon, plus athlétique. }\end{array}$ & $\begin{array}{l}\text { Armand, ouvrier à la chaîne d'une } \\
\text { usine de fabrication de raviolis, vit sa } \\
\text { routine métro-boulot-dodo dans une } \\
\text { grande barre d'HLM. Il va voir son } \\
\text { quotidien transformé par l'arrivée } \\
\text { d'un génie peu classique. Le génie, } \\
\text { tout droit sorti d'une boîte de } \\
\text { raviolis, va lui permettre de renouer } \\
\text { avec une vie bucolique grâce à deux } \\
\text { vœux. Le troisième vœu est réservé } \\
\text { aux pouvoirs non du génie mais à } \\
\text { ceux d'Armand... Ainsi, les deux amis } \\
\text { vont, semble-t-il, définitivement } \\
\text { rester ensemble «au vert " puisque le } \\
\text { spectateur les quitte en train de } \\
\text { chanter, les pieds dans l'eau. }\end{array}$ \\
\hline remarques & $\begin{array}{l}\text { Novélisation éponyme disponible de Jean- } \\
\text { Claude Carrière, illustrations de Pierre } \\
\text { Étaix, éditée chez Robert Laffont en 1958, } \\
\text { rééditée en } 2005 \text { et également rééditée en } \\
\text { littérature jeunesse en } 1985 \text { chez L'école } \\
\text { des loisirs. } \\
\text { Choix de la version initiale de } 1953 \text { et non } \\
\text { des versions revues et remontées par Tati } \\
\text { J. dans les années } 1960 \text { ou en } 1978 \text { car } \\
\text { c'est à partir de cette version que Jean- } \\
\text { Claude Carrière a novélisé le film. }\end{array}$ & $\begin{array}{l}\text { Adaptation de la bande dessinée } \\
\text { éponyme de Germano Zullo et } \\
\text { Albertine éditée en } 2002 \text { par La joie de } \\
\text { lire, Genève. } \\
\text { Ouvrage présent dans la sélection } \\
\text { bande dessinée de la liste d'ouvrages } \\
\text { recommandés par le Ministère de } \\
\text { l'Éducation Nationale pour le cycle } 3 \text {, } \\
\text { niveau de difficulté } 1 .\end{array}$ \\
\hline $\begin{array}{l}\text { questions et } \\
\text { présupposés }\end{array}$ & $\begin{array}{l}\text { Une séquence contraignante? } \\
\text { car éloignée des pratiques culturelles des } \\
\text { élèves }\end{array}$ & $\begin{array}{l}\text { Une séquence facilitante? } \\
\text { car proche de l'univers culturel de } \\
\text { référence des élèves }\end{array}$ \\
\hline
\end{tabular}




\subsubsection{Spécificités des séquences filmiques/supports d'écriture}

- Le film de Jacques Tati, Les Vacances de monsieur Hulot

24 présente une unité narrative en termes d'évènements (pratiquer l'équitation et ne pas y parvenir) et introduit des personnages très identifiables. Par ailleurs, cet épisode comporte deux temps distincts: un premier passage dans la maison de vacances de Martine et un deuxième temps sur la plage; Hulot peut donc déployer tous ses talents de grand maladroit: en privé et en solitaire mais aussi en public, comme bon nombre de scènes du film. Ainsi, l'inadaptabilité de Hulot au monde et aux réactions de son entourage ressort bien dans l'extrait, réunissant les deux facettes du personnage. On y trouve également les différents types de comique qui composent évidemment le film : comique de situation, comique de geste, comique de caractère (le comique de mots par définition étant absent puisque le film est pratiquement muet).

cernant le langage cinématographique et sa spécificité, les paramètres de ce langage fonctionnent pleinement dans ce film et dans la séquence choisie : les codes de la bande son et du montage sont mobilisés en tant qu'unités signifiantes, preuve en est le soin minutieux que Jacques Tati accordait à ces deux paramètres lors des postproductions au point même, concernant précisément Les Vacances de monsieur Hulot, de remonter plusieurs fois son long métrage après sa diffusion entre 1953 et 1978, et ce pour parvenir au film le plus abouti dans sa sélection de plans et dans l'ordre de succession de ceux-ci. Une attention scrupuleuse a évidemment été accordée aux effets visuels, aux cadrages, aux mouvements, à la lumière. La musique et la bande-son - qui est plus subtile qu'un simple bruitage - participent de l'atmosphère générale mais surtout de ce langage cinématographique où bande-son et bande-image se marient pour créer une langue à part, celle du cinématographe (Metz, 1968). Le traitement de la durée recouvre également son importance dans cette séquence où les ellipses et autres condensations temporelles sont de mise.

Dernier point notable: le traitement des personnages prend tout son sens par l'incarnation de l'acteur et la silhouette si célèbre d'Hulot nous rappelle combien l'acteur donne vie et corps au sens propre au personnage à l'instar de ce que les élèves, dans la novélisation, devront eux réaliser avec leur être de papier au moyen de matériaux purement linguistiques. 

inconvénient certain: la complexité de l'exercice pour les élèves. En effet, il y a finalement peu d'évènements, peu "d'action » à narrer qui serait susceptible de faciliter une mise en texte du film; les gags visuels à retranscrire constituent un obstacle du même ordre pour la textualisation, d'autant plus chez les jeunes élèves.

- Le film de Claude Barras, Le Génie de la boîte de raviolis

Pour contrebalancer la difficulté de la tâche précédente et disposer d'un étalon de comparaison, il a été décidé de soumettre les élèves à une deuxième passation de novélisation. Le court-métrage retenu est supposé facilitant par rapport au film de Jacques Tati. Sa complétude narrative - malgré une fin qu'on peut assimiler à une fin ouverte, son analogie avec un univers fictif de référence pour les élèves (le conte) l'ont d'emblée distingué. D'autres facteurs essentiels ont concouru au choix : genre similaire et durée équivalente à l'extrait de Tati mais dans une forme diégétique plus classique et familière aux élèves.

31 Les paramètres purement filmiques de film d'animation en couleurs et avec dialogues ont également présidé à sa sélection : il s'agit là d'une séquence vidéo narrative plus proche de l'ordinaire des habitudes de spectateur des élèves. Ce type de support est fréquenté par les élèves et sa lecture (ou spectature ${ }^{4}$ )-compréhension est accessible à un panel d'ampleur large d'élèves de 7 à 17 ans. Rappelons aussi que les novélisations auxquelles les jeunes élèves peuvent avoir affaire sont largement issues des dessins animés (Foot2rue, Le ranch, Inazuma eleven, Dora, etc.) d'où aussi l'élection d'un court métrage d'animation plutôt qu'un court métrage "classique». Cependant la technique de claymation ${ }^{5}$ rapproche considérablement la réalisation de ce court-métrage de celle d'un film en prise de vue réelle comme celui de J. Tatí. En outre, si l'on a souligné l'aspect plus classique et linéaire de la trame narrative de ce court métrage, ce dernier a largement été plébiscité par la critique et honoré par de nombreux prix pour le jeune public, soulignant ses qualités propres au médium cinéma ${ }^{7}$. L'œuvre source dont il est issu est elle-même préconisée par le ministère de l'Éducation nationale.

\section{Analyse des résultats}

\subsection{Méthodologie retenue}

L'entrée dans les productions des élèves a d'abord suivi une analyse relevant de la linguistique textuelle impliquant des marqueurs de cohérence et de cohésion (Charolles M., 1978 ; Bronckart, 1996). Cette entrée « classique » dans les textes des élèves nous a semblé pouvoir rendre compte de la gestion des textes par les élèves et aussi correspondre aux enjeux de l'enseignement de la production d'écrit à l'école, entendu que la cohérence vise de façon macrostructurelle la compréhensibilité de l'énoncé et son interprétation par le destinataire (notamment par le respect des métarègles de cohérence) et la cohésion assure au niveau microstructurel le bon fonctionnement de cet énoncé en permettant la transition de l'information d'un segment linguistique à un autre.

Une analyse narratologique a également été menée s'appuyant à la fois sur les catégories de G. Genette (1972) et le schéma de W. Labov et J. Waletzky (1967). Pour compléter l'observation des types narratifs et choix discursifs opérés par les élèves, les catégories d'A. Rabatel (2008) concernant le point de vue (PDV) qui croisent analyse narratologique 
et énonciative ont été mobilisées (point de vue représenté (montré), point de vue raconté (embryonnaire), point de vue asserté ainsi que la distinction énonciateur de premier ou de second plan).

- la clôture des textes ;

- les introductions de récit.

37 - caractéristiques énonciatives du texte (repérage des éléments qui signalent le positionnement adopté) :

- foyer narratif/ place du narrateur, en lien avec l'analyse syntagmatique de l'image ;

- le PDV (traces d'interprétation/de jugement, hiérarchie énonciateur/narrateur, phénomène de présence ou d'absence de sur-énonciation).

Ces indicateurs ont permis de hiérarchiser les productions selon le degré de réussite de la textualisation qui s'avère d'ailleurs en deçà du niveau attendu pour les élèves de fin de primaire $^{8}$. Néanmoins, ces marqueurs textuels seraient insuffisants à rendre compte des problèmes auxquels les élèves se sont confrontés pour transcoder sémiotiquement le langage cinématographique en langage écrit. Il semble donc utile d'aborder les écrits de ces élèves du point de vue du traitement du contenu diégétique en référence au déjà-là filmique : comment les élèves ont-ils transformé, digéré l'hypotexte filmique dans leur récit? Et évidemment dans cette droite ligne de la référence au substrat filmique initial, quel type de citation de cet hypotexte opèrent-ils?

La grille d'analyse suivra donc ces trois entrées principales: citation et références intertextuelles «filmiques", traitement du contenu diégétique et textualisation tout en croisant avec des tendances qui se dégagent chez les élèves en termes de profils de scripteurs.

Afin d'établir une typlogie de scripteurs « novélisateurs » pour l'instant absents du champ scolaire, il a été nécessaire d'avoir recours à des travaux sur des thématiques voisines qui traitaient également du déjà-là dans le discours des élèves. Ainsi, un transfert des observations menées par S. Plane, F. Rondelli et C. Venerin (2013) sur les stratégies des élèves au cours de la restitution par écrit de récits entendus a semblé opportun. Ainsi, nous avons largement emprunté à ces chercheuses leurs catégorisations de variations, fidélité et infidélité au substrat initial pour les appliquer au présent recueil de données. Cet éclairage a permis d'établir l'échelle de variabilité sur laquelle se positionne le scripteur pour rendre compte de l'intertexte filmique usant de toute l'amplitude de cette échelle : de la fidélité absolue au film à son infidélité la plus totale, voire à son dévoiement.

\subsection{Résultats : des profils diversifiés?}

1 Nous noterons tout d'abord l'annulation de l'hypothèse empirique de départ: la novélisation de la séquence vidéo du Génie de la boîte de raviolis n'a pas été mieux réussie 
que celle du film de Jacques Tati si l'on s'en tient à ce que les indicateurs retenus nous amènent à observer.

Cependant, au vu des premières analyses, ce deuxième recueil de productions nous renseigne sur d'autres stratégies d'écriture des élèves plus que sur un différentiel de difficultés entre les deux discours d'origine (les séquences filmiques). Il nous permet d'observer le traitement des dialogues et leur place au sein d'une re-narrativisation d'un déjà-là filmique. Il sert ainsi à mesurer en quelque sorte le degré d'investissement de l'élève dans son texte par rapport à la séquence source, à observer son positionnement: fidélité ou éloignement au déjà-là en croisant avec les autres critères de la grille d'analyse. Nous dénombrons six profils dans le corpus qui permettent de rendre compte de la diversité des traitements opérés par les élèves du déjà-là filmique et de décrire la variété de textualisation observée ainsi que les nuances de positionnement dans la relation de subordination à l'hypotexte. Nous les présenterons dans l'ordre du degré le plus fort de littéralité entre déjà-là filmique et discours écrit au degré le plus faible. Nous gardons évidemment à l'esprit que le transcodage sémiotique entre deux discours de nature différente constitue déjà une distance avec le langage du déjà-là filmique mais nous préserverons pourtant cette idée d'une volonté de fidélité «absolue» ou d'infidélité « marquée » dans les mécanismes de textualisation mis en œuvre par les élèves.

\subsubsection{Caractéristiques des profils et exemples}

44 Nous fournirons les éléments constitutifs de chacun des profils issus de l'analyse des textes des élèves et proposerons quelques extraits représentatifs de leur production. Néanmoins, il convient de préciser immédiatement que les productions des élèves se répertorient selon des tendances plutôt que selon des profils strictement délimités.

\subsubsection{Les profils de la fidélité : transcription, description, adaptation}

45 - Le profil transcription:

46 Dans ce profil, on note :

- l'absence du narrateur, une énonciation la plus neutre possible ;

- une fidélité quasi-totale à la séquence filmique (le contenu diégétique est transmis dans le texte autant que la mémoire puisse le permettre);

- de nombreux phénomènes de citations littérales du déjà-là (c'est le cas des dialogues par exemple, repris quasi à l'identique entre film et texte avec un phénomène de substitution du dialogue à la narration ou encore la volonté de retrouver mot à mot une formule magique non coutumière « froustri de froustra »).

Copie d'Enzo (CM1), (extrait), à partir des Vacances de monsieur Hulot :

il va dans le salon il vois un tableau de traver et puis il le remet après il done un coup de cane et il est encore de traver et puis il en vois un autre il le remer mes il en fait tomber un autre il vois un monsieur qui dort et après il sort de la maison et ouvre une porte et il $\mathrm{y}$ a un cheval blanc il $\mathrm{y}$ a une voiture qui a le coffre ouvère avec un monsieur dedans et le cheval done un cout sur le coffre et puis il se refferme il y a un monsieur qui est coinser dedans et puis le cheval s'en va et le monsieur se cache et le cheval le $\mathrm{r}$ retrouve Hulot et lui court après.

Dans cette production d'élève, outre les caractéristiques énoncées ci-dessus, on remarque également la disparition progressive de la ponctuation (début de copie non retranscrit) qui dénote un rapport de l'élève à son texte comme un déroulé continu des images qui 
défilent, les élèves n'ayant pas les clés pour analyser les marques d'enchainement à l'intérieur du discours filmique.

- Le profil description:

Copie de Sacha (CM2), (extrait), à partir du Génie de la boîte de raviolis :

Il était une fois une entreprise de raviolis. Un soir il était $18 \mathrm{~h} 30$ et un monsieur l'entreprise fermait tous les gens qui y travaillaient sortait et un monsieur alla à la gare pour prendre le train, il rentra chez lui, et ouvra la fenêtre de son appartement. Il arosa une fleur et la regarda. Il referma la fenêtre et il ouvri un placard que de boîte de raviolis et il en pris une. Il l'ouvra et un génie sortit de la boîte et il se mit à chanter. Puis il dit « tu as le droit à deux vœux » [...]

51

Les éléments constitutifs de ce type de profil sont :

- la présence d'un narrateur a minima : la focalisation externe est privilégiée, utilisée comme marque de témoignage (le lecteur lit le texte comme le spectateur a vu le film avec l'illusion de la caméra objective) ;

- les plans de la séquence filmique sont restitués sans hiérarchisation visible dans leur successivité initiale de l'hypotexte filmique; le scripteur suit linéairement la diégèse filmique, la citation prend la forme d'un calque à l'écrit du discours filmique ;

- on peut parler d'une pseudo-narration avec projection d'un lecteur-spectateur dans le texte dans la mesure où le souci de la textualisation ne prévaut pas sur la restitution des évènements du déjà-là filmique.

Dans ces deux profils, on observe donc la transcription littérale de la suite des images, de la séquence filmique à la manière d'un déroulé fidèle à la séquence vidéo sans ajout, ou très à la marge. Les tentatives de textualisation concernant les CM1-CM2 restent maladroites avec très peu de reprises anaphoriques, une absence assez remarquable de connecteurs temporels et logiques.

53 - Le profil adaptation

54 Dans ce profil, une attention accrue est portée à la mise en place de la trame narrative : le traitement diégétique opéré par le scripteur ne se résume pas à la restitution littérale des plans de la séquence vidéo. Des tentatives d'organisation interne du texte apparaissent avec:

- un cadrage a minima ;

- une orientation (résumé de l'action).

Néanmoins, le scripteur persiste dans une attitude de conformité au déjà-là filmique par le respect assez strict de la linéarité du discours filmique et du traitement temporel de la diégèse filmique.

L'exemple ci-dessous d'incipit d'un texte de première illustre la volonté de mettre en place la situation, volonté absente des profils précédents qui débutaient leur narration à l'identique du déjà-là filmique sans médiation préalable ni présentation du contexte par le narrateur.

57 Copie d'Emma (1 $\left.{ }^{\mathrm{r}}\right)$, (extrait), à partir des Vacances de monsieur Hulot:

Le mois d'août fut très chaud cette année là. Monsieur Hulot, vêtu tel un véritable cavalier alla sonner à la porte de son amie, Martine, afin de l'emmener faire une balade à cheval sous un beau soleil d'été. La domestique lui ordonna de patienter dans le salon le temps que son amie se prépare. 


\subsubsection{Les profils de la variation et de l'infidélité : appropriation, invention, re-création}

\section{l'intégralité de la séquence filmique non respectée.} novélisé ; souhaitée et simulée des profils précédents ; camper les personnages.

- Variations et infidélité : dans le profil invention diégétique du déjà-là filmique.

- établir une organisation concertée de la narration avec un cadrage, une orientation, des résumés, le scripteur fait figurer des commentaires narratifs dans son texte et des traces d'interprétation du discours filmique transparaissent dans le texte second, l'hypertexte

- choisir un point de vue qui tranche avec la focalisation extérieure et son extériorité

- opérer des réductions et expansions des durées de la séquence vidéo afin de conférer à son texte une cohérence et un ordonnancement réfléchi (hiérarchisation des évènements du déjà-là filmique entre eux et évaluation de leur importance pour les insérer dans le texte novélisé, en variant leur niveau de traitement de l'hypotexte à l'hypertexte).

Une fois encore l'incipit des Vacances de monsieur Hulot est révélateur des procédures de ce profil : le texte ci-dessous d'une élève de première présente une expansion forte du plan initial de la séquence filmique qui ne dure que quinze secondes (sur sept minutes trente). Ce passage anecdotique pour beaucoup d'élèves et éludé dans la plupart des cas est ici largement amplifié pour asseoir la narration de l'auteur; le scripteur mobilise les potentialités du point de vue interne qui fait découvrir le discours intérieur des personnages et donc leur mobile, il inscrit des indices qui anticipent la scène à venir sur la plage et le duel entre Hulot et sa monture, il déploie une description précise pour

\section{Copie de Capucine ( $\left.1^{\text {re }}\right)$, (extrait), à partir des Vacances de monsieur Hulot :}

Monsieur Hulot sentit, dès le premier contact, qu'il n'aimait pas les chevaux et que les chevaux ne l'aimaient pas non plus. Pourtant, cela avait été furtif, à peine quelques secondes. Mais un frisson était partir de sa tête et avait traversé son cou, ses épaules, sa poitrine, ses cuisses pour finir jusque dans la plante de ses pieds. Les chevaux, eux, surtout le blanc qui paraissait particulièrement fourbe, avaient effectué un mouvement de recul, en levant et en abaissant la tête, les yeux presque révulsés. S'il avait été tout à fait seul, Monsieur Hulot aurait tourné les talons et serait parti en courant. Il aurait peut-être même battu le nouveau record du cent mètres, malgré ces grandes bottes de cuir qui le génaient. Oui, c'est sûrement ce qu'il se serait passé s'il n'y avait pas eu Martine. Martine et ses longues jambes, Martine et ses cheveux bouclés et dorés, Martine et son parfum de jasmin... C'est pourquoi, au lieu de fuir, le plus loin possible, Monsieur Hulot parcouru d'un pas énergique pour se donner une contenance, les quelques mètres qui le séparaient de la maison de la jeune femme. Mais arrivé sur le perron, première chute. [...]

La citation est certes présente mais avec un niveau élevé de liberté prise non seulement par rapport à la chronologie des évènements de l'hypotexte mais aussi par rapport à

Ainsi des choix d'ellipses raisonnées participent à l'organisation de la narration pleinement planifiée par le scripteur pour créer des effets stylistiques, intentionnels de la part de l'élève-auteur. 
- une invention presque totale manifestée par le non-respect des évènements ou de la trame narrative de l'hypotexte filmique ;

- une citation minimale (reprise des personnages, éventuellement du cadre global de la diégèse). 
quitter cet endroit et trouver un endroit magnifique, une clairière bordée d'arbres, des papillons s'envolant dès que je m'approcherai d'eux. Je voulais suivre le chemin de mon frère Valek partir de cette ville, échapper au travail dans l'usine de raviolli à refermer des boites avec oncle Armand. [...] posé sur le rebord de ma fenêtre un magnifique papillon sculté dans de l'ébène accroché à une chaîne en argent attendait. Un mot était coincé en-dessous :

«Un lieu comme tu le souhaite t'attends,

le vent s'ouffe sur les milliers d'arbres de la forêt,

des papillons buttinent les fleurs par centaines.

Rejoins - moi petit papillon, un seul papillon manque à tout ça »

Mon cœur fit un bond seul Valek connaissait mon rêve, et on s'était promit de le réaliser ensemble [...] je rejoindrais ce lieu où je pourrais faire mille accrobatie ou je pourrais monter aux arbres et me déplacer de branches en branches comme je le faisais chez mamie Ambroise quand j'étais petite. Finis les repas que de raviolis [...]. Mais avant je devais en parler Sam et Jules, on partirait ensemble, nous formons et formerons les trio inséparables. Le collier avec le papillon sculté est le symbole d'une nouvelle vie et d'un rêve qui sera accomplit, dans quelques jours je quitterais tout ça pour une vie que j'espère meilleur. au côté de mes amis Sam et Jules alias Julianna et de mon frère ainsi que de ces deux amis qui a rencontré Ari et Janco.

Dans cette production, le personnage principal Armand devient largement secondaire, le Génie est même supprimé, des personnages-héros sont rajoutés au risque de la confusion. Le texte conserve en arrière-plan le contexte routinier qui devient le mobile de la fuite du nouveau personnage principal, fuite mise en scène dans un univers qui tient plus du récit d'aventures que du merveilleux, genre dans lequel s'inscrivait l'hypotexte. Il y a donc déplacement de registre avec préservation des décors (la clairière, la toile de fond « des raviolis ») pour tendre la diégèse vers le roman d'apprentissage-initiatique adolescent.

\subsubsection{Synthèse rapide sur l'analyse globale}

Les six profils sont perceptibles mais leurs frontières sont non étanches, les élèves oscillent souvent entre deux tendances et quatre catégories majeures se profilent pour classifier les productions d'élèves.

- Transcription-description

- Description-adaptation

- Appropriation-invention

- Invention-(re-)création

Ces catégories viennent confirmer la posture du scripteur par rapport au déjà-là filmique : même lorsque les productions superposent plusieurs profils, le fonctionnement interne de ces productions est en congruence avec une démarche de référenciation à l'hypotexte ( fidélité, variation ou infidélité).

77 Par ailleurs, à la fois en CM1-CM2 et dans la classe de première, on retrouve les différents profils ou des traces de ces profils via des segments de texte ou des microéléments textuels pour les élèves les plus jeunes à l'exception de la re-création exclusive de la classe de première.

Cependant des tendances se dessinent dans les deux groupes :

- En CM1-CM2, les profils sont majoritairement de l'ordre de la transcription-description.

- En première, le profil appropriation/invention prend le pas sur les autres avec des traces d'adaptation et de description. Les productions sont néanmoins plus marquées et leur classement en type de profil est plus restrictif. Les élèves de première tiennent une ligne de 
mise en texte "novélisante » plus stricte et exclusive que les élèves de primaire dont la variété et l'instabilité dans les profils amènent à une complexité d'analyse. classe avec des élèves qu'on imagine plutôt habitués à une séquence didactique qu'à une mise en situation "brute» telle que le recueil a été mené. Cependant, le fait que les conditions soient écologiques nous permet de supposer que les procédures mobilisées ordinairement par les élèves dans des situations d'écriture en classe ont opéré. Ainsi, nous constatons que suivant leur niveau et leur habileté, nos élèves ont mobilisé prioritairement deux modalités d'investissement du texte novélisé :

- les CM1-CM2 sont clairement dans le relater au détriment du narrer (qualité de la textualisation à l'appui),

- les premières tentent de mettre en jeu la langue, sans négliger le narrer bien entendu, et adoptent ainsi une posture auctoriale vis-à-vis de leur texte ; ils dépassent l'exercice scolaire pour affirmer une inventio qui va au-delà de la novélisation la plus répandue, celle de premier degré.

81 Pour conclure provisoirement, nous pouvons avancer que les élèves ont réalisé avec le profil invention des novélisations de second degré. L'élève qui adopte ce profil joue avec l'intertexte filmique et « digère » le déjà-là filmique pour faire œuvre nouvelle, il assimile le film sans le plagier ni le copier, en s'éloignant aussi d'une traduction-adaptation qui resterait fidèle à la littéralité de la diégèse. En ce sens, les élèves de ce profil accomplissent les virtualités paradigmatiques du langage filmique que le film en tant qu'objet de discours et œuvre unique ne peut offrir au spectateur. Le film devient levier et point de départ d'un texte nouveau ; il est aussi enjeu du récit et de la narrativité car il est mobilisé en tant qu'hypotexte central par l'auteur mais doit être absolument connu du lecteur pour que ce dernier goûte tout le sens de la variation autour de la source, du déjàlà filmique.

\subsection{Tendances majeures et cas de profil saillant : explications}

\subsubsection{Le profil le plus représenté au CM1-CM2}

Nous avons mentionné que les élèves de CM1-CM2 se situent de façon massive sur les profils transcription et description et le recours à cette filiation forte à l'hypotexte peut trouver son origine dans l'illusion référentielle instaurée par la bande-image du film. Les élèves ont été captivés (pour user de la polysémie du terme) par la dimension déictique du film et ont occulté en quelque sorte sa dimension diégétique. Ils retranscrivent à l'écrit ce qu'ils ont vu, refusant de prendre en charge la narration de leur propre texte qui doit montrer, doit ressembler le plus à ce qu'ils ont vu du film, ce qu'ils en ont capturé en tant que bande-image et qu'ils n'ont pas analysé comme une construction discursive. Ils ne renarrativisent donc pas le déjà-là filmique et le vident presque de sa dimension narrative en ne restituant qu'une suite de plans sans recréer d'intentionnalité ou de fonction thymique à leur récit écrit. 


\section{planification (Hayes \& Flower, 1980) comme on aurait pu s'y attendre puisque le synopsis d'un récit est mis à disposition grâce à l'intertexte filmique.}

dans un stade de l'ordre du pré-narratif au lieu de le délester des opérations de

\subsubsection{Un profil atypique : le cas de la re-création en classe de première}

Un trait saillant ressort au niveau de la classe de première : certains élèves abordent le déjà-là filmique comme un espace de très grande liberté à l'inverse des élèves de fin de primaire qui le conçoivent comme une contrainte. Dans le cas de la re-création, le texte produit n'a plus qu'un rapport très éloigné avec la séquence filmique, voire il s'en est complètement affranchi ; le film est une source d'inspiration du texte de l'élève au même titre que d'autres sources de création, l'hypotexte filmique n'est qu'un déjà-là parmi d'autres, non privilégié, le texte produit devient un texte autonome qui ne suppose pas de lien direct avec le film. La re-création est vraisemblablement un moyen d'échapper au genre spécifique qu'est la novélisation et de renouer avec une tradition d'écriture libre.

Citons ce début de production de première emblématique :

Copie de Virginie $\left(1^{\mathrm{re}}\right)$, (extrait), à partir des Vacances de monsieur Hulot :

Alors voilà, c'était comme ça qu'il allait finir. Lui, Victor Hulot, né de père Daniel Hulot et de mère Catherine Hulot, allait se suicider. Pathétique n'est-ce-pas? Mais bon il fallait positiver, il ne laisserait pas un grand vide chez les siens, ils y étaient habitués à ses fantaisies; ses sautes d'humeur perpétuelles. Il aurait été presque dommage qu'il meurt normalement, ça aurait déçu. En plus, il trouvait que ça sonnait bien, sa fin tragique avec Charlène en pleures près de son corps inerte; « elle aurait au moins servi à quelque chose celle-là pensait-il en souriant, parce que çà hein... Cela faisait exactement trente minutes qu'il l'attendait et l'air de circonstance qu'il s'était donné en arrivant avait fondu au fur et à mesure que le temps passait, découvrant l'appartement sale et délabré [...]

Il s'agit ici d'un exemple extrême mais qui illustre bien le procédé mis en œuvre : dans le cas présenté, Hulot veut se suicider à la fin de la scène. L'élève transforme le film comique en une tragédie personnelle puisque tout est raconté du point de vue interne de Hulot; les intentions du film sont complètement réorientées voire dévoyés et en ce sens, le texte novelisé devient indépendant du film, le déjà-là filmique devient même un prétexte à la création du texte du scripteur. L'intentionnalité portée par le texte se désolidarise de celle de l'auteur du film.

\section{Conclusion}

88 Le travail présenté ici mérite encore des prolongements en recherche. Néanmoins ce recueil exploratoire auprès d'élèves de niveaux divers a déjà permis de mettre à jour un certain nombre de procédures utilisées par les élèves et de déterminer des profils de scripteurs qui ont maille à partir avec le déjà-là filmique. Ce matériau exogène est complexe à gérer pour les scripteurs car il devrait normalement les obliger à réfléchir dans deux systèmes de langages distincts, tâche éminemment compliquée à gérer. Pourtant, il peut aussi représenter un formidable espace disponible pour la textualisation, riche de variations pour le jeune scripteur qui s'y prête.

Les perspectives didactiques offertes par le genre sont désormais ouvertes. Et elles apparaissent prometteuses au regard de premiers dispositifs expérimentaux mis en place 
en classe de fin de primaire et dont les résultats sont en cours de traitement. Ainsi, la deuxième phase du protocole menée récemment (juin 2015) et centrée cette fois sur un dispositif didactique autour du genre de la novélisation confirme les profils de la première phase exploratoire exposée ici. Cependant, l'évolution des profils s'avère bien plus nette lors de cette deuxième phase et surtout, les caractéristiques propres au genre novélisation et si particulières à la rencontre de deux langages distincts semblent fonctionner comme des leviers précieux pour faire entrer les élèves dans des processus d'écriture complexes.

\section{BIBLIOGRAPHIE}

BAETENS, J. (2006). «La novellisation contemporaine en langue française ». Fabula LHT 2. En ligne : http://www.fabula.org/lht/2/baetens.html. - (2008). La novellisation. Du film au roman. Lectures et analyses d'un genre hybride, Bruxelles : Les Impressions nouvelles.

BAKHTINE, M. (1984) [1979]. Esthétique de la création verbale. Trad. du russe par Alfreda Aucouturier, Paris : Gallimard.

BESSON, R., (2014). « Prolégomènes pour une définition de l'intermédialité ». Cinémadoc. En ligne : http://cinemadoc.hypotheses.org/2855.

BOURHIS, V. \& ALLAIN-LE FORESTIER, L. (2010). «Le conte novélisé : un nouveau genre textuel ? L'exemple de Kirikou, de Michel Ocelot ». Synergies France 7, p. 127-134.

BOURHIS, V. \& LE CORFF, I. (2013). « Kirikou et la sorcière, de l'écran à l'encre ». Interférences littéraires/Literaire interferenties 11, p. 115-129.

BRONCKART, J.-P. (1996). Activité langagière, textes et discours. Neuchâtel : Delachaux et Niestlé. CAROU, A. (2004). «Cinéma narratif et culture littéraire de masse : une médiation fondatrice (1908-1928)». Revue d'histoire moderne et contemporaine 51(4), p. 21-38.

CHAROLLES, M. (1978). « Introduction aux problèmes de la cohérence des textes ». Langue française 38, p. $7-41$

Compagnon, A. (1979). La Seconde Main ou le travail de la citation. Paris : Seuil.

- (1998). Le Démon de la théorie. Littérature et sens commun. Paris : Seuil.

CRINON, J. (2006). «L'écriture littéraire et les genres ». Le français aujourd'hui 153, p. 17-24.

DOLZ-MESTRE, J. \& GAGNON, R. (2008). « Le genre du texte, un outil didactique pour développer le langage oral et écrit ». Pratiques 137/138, p. 179-198.

FERRIER, B. (2006). « Les novélisations pour la jeunesse : reformulations littéraires du cinéma ou reformulations cinématographiques de la littérature ? ». Fabula LHT 2. En ligne : http:// www.fabula.org/lht/2/Ferrier.html. 
GENETTE, G. (1972). Figures III. Paris : Seuil.

- (1979). Introduction à l'architexte. Paris : Seuil.

- (1982). Palimpsestes : la littérature au second degré. Paris : Seuil.

haltÉ, J.-M., Petitjean, A. \& PLANE, S. (2002). « L'écriture et son apprentissage ». Pratiques 115-116, p. 1-253.

HAYES, J. R. \& FLOWER, L. (1980). « Identifying the Organization of Writing Processes ». In : Gregg, L.-W. \& Steinberg E. R., Cognitive Processes in Writing. Hillsdale. New Jersey : L. Erlbaum, p. 3-30.

JAUSS, H. R. (1978). Pour une esthétique de la réception. Paris : Gallimard.

KERN, S. \& RAFFARA, A. (2012). « Effet du type de support imagé sur la production du récit chez l'enfant ». In : Delamotte, R. \& Akinci, M.-A. (dir.), Récits d'enfants. Développement, genre, contexte. Rouen : Presses universitaires de Rouen et du Havre, p. 97-115.

KRISTEVA, J. (1969). Sémiotikè, recherches pour une sémanalyse. Paris : Seuil.

LABOV, W. \& WALETZKY, J. (1967). « Narrative analysis ». In : Helm, J. (éd.), Essays on the Verbal and Visual Arts. Seattle : U. of Washington Press, p. 12-44.

LACELLE, N. (2009). Modèle de lecture-spectature, à intention didactique, de l'œuvre littéraire et de son adaptation filmique. Thèse de doctorat : Université du Québec à Montréal. En ligne : http:// www.archipel.uqam.ca/2537/.

LEFEBVRE, M. (2007). « Le parti pris de la spectature ». In : Gervais, B. \& Bouvet, R. (dirs), Théories et pratiques de la lecture littéraire. Montréal, Presses de l'Université du Québec, p. 225-270.

METZ, C. (1966). « La grande syntagmatique du film narratif ». Communications 8, p. 120-124.

METZ, C. (1968). Essais sur la signification au cinéma, tome I. Paris : Klincksieck.

PASOLINI, P. (1966). « Le scénario comme structure tendant vers une autre structure ». Cahiers du cinéma 185, p. 76-83.

PLANE, S. (2003). « Stratégies de réécriture et gestion des contraintes d'écriture par des élèves de l'école élémentaire : ce que nous apprennent des écrits d'enfants sur l'écriture ». Rivista Italiana de Psicolinguistica Applicata III/1, p. 57-77.

- (2006a). « L'écriture de fiction existe-t-elle ? ». Repères 33, p. 7-19.

- (2006b). «Singularités et constantes de la production d'écrit. L'écriture comme traitement de contraintes ». In : Laffont-Terranova, J. \& Colin, D., Didactique de l'écrit. La construction des savoirs et le sujet-écrivant. Namur : Presses universitaires de Namur, p. 33-54.

PLANE, S., RONDELLI, F. \& VENERIN, C. (2013). « Variations, fidélité, infidélité : l'écriture et la réécriture de discours rapportés par de jeunes scripteurs ». In : Desoutter, C. \& Meilet, C. (dirs), Le discours rapporté : approches linguistiques et perspectives didactiques. Berne : P. Lang, p. 215-232.

RABATEL, A. (2008). Homo Narrans, pour une analyse énonciative et interactionnelle du récit. Limoges : Lambert-Lucas.

RICeUR, P. (1991a). Temps et récit, tome 2, la configuration dans le récit de fiction. Paris : Seuil.

- (1991b). Temps et récit, tome 3, le temps raconté. Paris : Seuil.

SCHAEFFER, J.-M. (1989). Qu'est-ce qu'un genre littéraire ? Paris : Seuil. 


\section{Littérature primaire}

CARRIÈRE, J.-C. (1985). Les Vacances de monsieur Hulot : d'après le film de Jacques Tati. Paris :

L’École des loisirs.

JAILLET, N. (2011). La nouvelle guerre des boutons : un film de Christophe Barratier. Paris :

Hachette.

La guerre des boutons (Robert, 1962).

Le génie de la boîte de raviolis (Barras, 2005).

Les parapluies de Cherbourg (Demy, 1964).

Les vacances de Monsieur Hulot (Tati, 1593).

PERGAUD, L. (1981). La guerre des boutons. Paris : Gallimard.

ROSENTHAL, O. (2012). Ils ne sont pour rien dans mes larmes. Paris : Gallimard.

Vertigo (Hitchcock, 1958)

VIEL, T. (1999). Cinéma. Paris : Minuit.

zULLO, G. \& ALBERTINE (2002). Le génie de la boite de raviolis. Genève : La Joie de lire.

\section{NOTES}

1. Voir à ce sujet, l'article très documenté d'A. Carou (2004, p. 21-38).

2. C'est le cas de Cinéma de Tanguy Viel ou de For ever Mozart de Jean-Luc Godard.

3. Pour une synthèse sur la notion, on peut notamment consulter Prolégomènes pour une définition de l'intermédialité publié le 29 avril 2014 sur Cinémadoc, hébergé par hypothèses.org, un texte de Rémy Besson, (2014) postdoctorant à l'université de Toulouse, laboratoire commun RiMeC, programme ANR, LLA-CREATIS.

4. Sur la notion de spectature en relation avec la lecture compréhension-interprétation, voir les travaux des québécois Martin Lefebvre (2007) et Nathalie Lacelle (2009).

5. Animation de structures en pâte à modeler.

6. L'obligation de filmer avec une caméra les scènes du film d'animation en trois dimensions amène le réalisateur à composer avec les mêmes éléments que ceux de la prise de vue réelle : éclairage, mouvements de caméra, choix de l'objectif, profondeur de champ.

7. Prix du Public \& Prix du Meilleur Film d'Animation, 41 ${ }^{\mathrm{e}}$ Journées de Soleure, Suisse, 2006 ; Prix Kodak du Public, Festival Cinématou, Genève, 2006; Golden Spotlight Award, Festival de Melbourne, 2006 ; International Newcomer in Animation Award, Bristol Brief encounters, 2006 ; $35 \mathrm{~mm}$ short film Prize, Circuito Santiago de Compostela, 2006.

8. Au regard des compétences attendues au palier 2 du livret de compétences de l'élève en vigueur au moment de l'expérimentation mais également en considération des récentes propositions faites dans les nouveaux programmes pour les cycles 2 et 3 (B.O. du 26 novembre 2015). 


\section{RÉSUMÉS}

Cet article se propose d'étudier l'investissement d'un genre de discours, la novélisation, par des élèves de l'école élémentaire et secondaire, genre pleinement ancré dans la problématique du déjà-là puisque, par définition, il met en œuvre la transformation d'un matériau initial, celui du récit filmique. Il s'agira d'abord de définir brièvement la novélisation à partir de son histoire et de ses caractéristiques génériques qui le fondent en un objet hybride, entre deux langages. Puis, au travers de la présentation d'une recherche en cours impliquant plusieurs classes de niveaux divers dont les élèves ont été soumis à l'exercice de style que constitue la novélisation, nous observerons comment ces derniers résolvent les problèmes qu'elle pose en tant que genre tributaire d'une source pré-existante. Enfin, à partir de l'analyse des stratégies rédactionnelles déployées par les élèves dans leurs productions, nous esquisserons une typologie de positionnements auctoriaux qui traduisent des procédures spécifiques pour traiter du genre et de la représentation que les élèves s'en font.

This article will examine the use of a particular speech genre - novelization - by primary and secondary school pupils, a genre fully rooted in the issue of the pre-existing, since by definition, it implements the transformation of an initial material, that of film narrative. First, this will aim to shortly define novelization from its history and its characteristics making it something hybrid, between two languages. Then, via the presentation of an ongoing research involving several multi-level classes, the pupils of which have been given a novelization exercise, we will study how they solve the problems novelization can cause as a genre depending on a pre-existing source. Finally, from an analysis of the writing strategies used in those pupils' productions, we will outline a classification of the various auctorial positions which point out the specific procedures used to deal with that particular genre and the perception the pupils have of it.

\section{INDEX}

Mots-clés : novélisation, textualisation, profils de scripteurs, déjà-là filmique

Keywords : novelization, textualization, scriptwriters' profiles, pre-existing films

\section{AUTEUR}

\section{SONIA CASTAGNET-CAIGNEC}

Université de Paris-Sorbonne/Université de Caen Normandie, STIH/ESPÉ, F-14000 